\title{
Corrigendum to "Ion cyclotron waves during the Rosetta approach phase: a magnetic estimate of cometary outgassing" published in Ann. Geophys., 31, 2201-2206, 2013
}

\author{
M. Volwerk ${ }^{1}$, C. Koenders ${ }^{2}$, M. Delva ${ }^{1}$, I. Richter ${ }^{2}$, K. Schwingenschuh ${ }^{1}$, M. S. Bentley ${ }^{1}$, and K.-H. Glassmeier ${ }^{2}$ \\ ${ }^{1}$ Space Research Institute, Austrian Academy of Sciences, 8042 Graz, Austria \\ ${ }^{2}$ Institute for Geophysics and Extraterrestrial Physics, TU Braunschweig, Germany
}

Correspondence to: M. Volwerk (martin.volwerk@oeaw.ac.at)

In the paper by Volwerk et al. (2013) it reads on page 2203, left column, seventh line from the bottom: "This means that the ring distribution could have been fully scattered into a shell distribution ..."

There is, however, a typo in this sentence. The correct version should read as follows: "This means that the ring distribution could not have been fully scattered into a shell distribution ..."

\section{References}

Volwerk, M., Koenders, C., Delva, M., Richter, I., Schwingenschuh, K., Bentley, M. S., and Glassmeier, K.-H.: Ion cyclotron waves during the Rosetta approach phase: a magnetic estimate of cometary outgassing, Ann. Geophys., 31, 2201-2206, doi:10.5194/angeo-31-2201-2013, 2013. 